\title{
Physiologic Basis and Pathophysiologic Implications of the Diastolic Properties of the Cardiac Muscle
}

\author{
João Ferreira-Martins and Adelino F. Leite-Moreira \\ Department of Physiology, Faculty of Medicine, University of Porto, Alameda Prof. Hernâni Monteiro, 4200-319 Porto, Portugal \\ Correspondence should be addressed to Adelino F. Leite-Moreira, amoreira@med.up.pt
}

Received 1 November 2009; Revised 15 February 2010; Accepted 21 March 2010

Academic Editor: Henk L. M. Granzier

Copyright () 2010 J. Ferreira-Martins and A. F. Leite-Moreira. This is an open access article distributed under the Creative Commons Attribution License, which permits unrestricted use, distribution, and reproduction in any medium, provided the original work is properly cited.

\begin{abstract}
Although systole was for long considered the core of cardiac function, hemodynamic performance is evenly dependent on appropriate systolic and diastolic functions. The recognition that isolated diastolic dysfunction is the major culprit for approximately fifty percent of all heart failure cases imposes a deeper understanding of its underlying mechanisms so that better diagnostic and therapeutic strategies can be designed. Risk factors leading to diastolic dysfunction affect myocardial relaxation and/or its material properties by disrupting the homeostasis of cardiomyocytes as well as their relation with surrounding matrix and vascular structures. As a consequence, slower ventricular relaxation and higher myocardial stiffness may result in higher ventricular filling pressures and in the risk of hemodynamic decompensation. Thus, determining the mechanisms of diastolic function and their implications in the pathophysiology of heart failure with normal ejection fraction has become a prominent field in basic and clinical research.
\end{abstract}

\section{Introduction}

From the earliest stages of an individual's life, the heart has the challenging mission to uninterruptedly transfer its kinetic energy to the blood. This task is accomplished by means of its intrinsic mechanical activity, which can be considered as being composed of two continuous and interdependent functions: systole and diastole. Being a living structure with biomechanical sensing features [1] the heart is a paradigmatic organ of continuous mechanical adaptation [2]. In this way, it is able to adapt its intrinsic mechanical properties when different loads and neurohumoral conditions are imposed so that new mechanical and biological steady states may be eventually achieved $[1,3,4]$. However, permanent pathologic stimuli make this equilibrium at best, short-termed, imposing an imbalance towards a progressive impairment of cardiac function and inexorably leading to a dysfunctional phenotype. This process consists of both intraand extracellular remodeling, with deleterious consequences at structural, molecular, and functional levels.

Despite the knowledge that the heart spends almost two-thirds of its time in diastole (relaxing and filling), its contractile activity was for a long time considered the core of its mechanical function and over which major concerns had been focused. Indeed, it was not until the nineteen eighties that the scientific community began to realize the clinical significance of diastolic dysfunction among patients with signs and symptoms of heart failure (HF) but in whom ejection fraction was rather preserved. The recognition of the latter condition as "heart failure with normal ejection fraction" (HFNEF) impelled major efforts in order to identify the pathophysiological mechanisms underlying this emerging concept. Female gender, older age, arterial hypertension, diabetes, obesity, and left ventricular (LV) hypertrophy are currently well-established risk factors [5-21], showing a strong association with impaired diastolic function with a concomitant normal or only mildly abnormal systolic function. However, and according to the Heart Failure and Echocardiography Associations of the European Society of Cardiology, these patients are only classified as having HFNEF if they develop signs or symptoms of HF along with the objective recognition of normal systolic LV function and of diastolic LV dysfunction [22]. Deserving special relief is the fact that the therapeutic management of HFNEF is still 
relying on an empirical framework and is not translating into any survival improvement in this particular group of patients. However, it is currently not clear whether these neutral outcomes are due to specific mechanisms in the pathophysiology of HFNEF and/or to the nonadherence of clinical trials to the existing diagnostic guidelines of HFNEF. The latter has been recently shown to be responsible for a high variability among the recruited patients in HFNEF trials and therefore in their reported final results $[23,24]$. Importantly, a deeper understanding of the mechanisms leading to the development of HFNEF as well as a systematic compliance of the clinical trials to the current guidelines is seriously warranted, setting the necessary conditions for an efficient cross-talk between clinical and basic research.

\section{Risk Factors, Diastolic Function, and HFNEF}

It has been documented that LV structure and function differ between HFNEF and HFREF [25], which may depend on the relative impact that associated clinical conditions have on the development of a particular cardiac phenotype or disease. Regarding HFNEF, female gender seems to be a major risk factor for its development [16-20], not only by being more frequently associated with other risk factors (e.g., arterial hypertension) but also by rendering the postmenopausal heart more susceptible to the remodeling features associated with slower relaxation and lower ventricular compliance. Even if the first studies indicating a higher risk in females $[17,26]$ have missed an objective evaluation of diastolic dysfunction among the diagnosed cases, the conclusion that such a trend may actually exist got early support from clinical cardiologists. Indeed, recent epidemiological data from the Framingham Heart Study [27] showed that within the HF population studied (median age of HF onset of 78 years old), individuals with HFNEF were more likely to be women (65\% women versus 35\% men), with an ageadjusted odds ratio of 2.55 when compared to men. Arterial hypertension or valvular heart disease also duplicated the risk of developing HFNEF and both risk factors were more frequent in women as well. Although this study showed that survival was similar between genders and independent from left ventricular ejection fraction (LVEF), results from the CHARM program [28] indicated that women had a better survival than men, even after adjustment for cause of heart failure, LVEF, and age.

All these cumulative epidemiological data supporting gender specificities in the risk of diastolic dysfunction and HFNEF impelled basic research to study the mechanisms underlying such differences, whose knowledge may contribute to design more specific therapeutic targets and guidelines for HF in general and HFNEF in particular. In this regard, $17 \beta$-estradiol is a distinctive feature between genders and has been shown to be an important modulator of myocardial relaxation and passive properties [16]. Considering cardiomyocyte $\mathrm{Ca}^{2+}$-handling and relaxation, $17 \beta$-estradiol modulates the expression and activity of Ltype $\mathrm{Ca}^{2+}$ channels [29], the phosphorylation levels of PLB [30], myofilaments $\mathrm{Ca}^{2+}$ sensitivity [31] and protects single cardiomyocytes against $\mathrm{Ca}^{2+}$ loading induced by hypoxia [32]. Important mediators of $17 \beta$-estradiol within the cardiovascular system and with recognized benefits on diastolic function are nitric oxide (NO) and endogenous brain natriuretic peptide (BNP) whose levels are also higher in females than in males [16]. By promoting the actions of these neurohumoral agents, estrogens may increase the speed of relaxation and ventricular compliance, improving overall diastolic function. Beyond these acute and indirect effects, estrogens also modify myocardial material properties by reducing cardiac fibroblast proliferation, collagen turnover and pressure overload-induced cardiomyocyte hypertrophy $[33,34]$. Therefore, it seems reasonable to speculate that the loss of these beneficial effects after menopause may render the female heart especially vulnerable to diastolic dysfunction. Of special remark, the absence of the long-term protective influences of estrogens confers a higher susceptibility of the female heart to the risk factors commonly leading to HFNEF, such as obesity, hypertension, and diabetes [35, 36]. Actually, comparing individuals from both genders with similar degrees of arterial hypertension or aortic stenosis, women develop more often concentric cardiac remodeling while males develop more often eccentric remodeling [35]. As concentric hypertrophy leads to increased wall thickness and possibly smaller chambers, the resulting ventricular geometry leads to earlier partial chamber filling, with increases in operating stiffness [16]. The synergic effects of obesity and hypertension to the development of left ventricular hypertrophy are much stronger in women than in men as is greater the effect of diabetes to the development of congestive HF in the female gender [37].

Independently or in association, all these risk factors may cause structural and functional changes at the organ, cellular and molecular levels of the heart, leading to a slower relaxation and/or increased chamber stiffness. A detailed discussion on these determinants of diastolic function follows.

\section{Diastolic Properties of the Cardiac Muscle-A Composite Material}

Cardiac muscle tissue is a composite material consisting of cardiomyocytes, fibroblasts, blood vessels, and extracellular matrix (ECM). Therefore, it is reasonable to postulate that changes in any of the elements within the myocardium may affect its material properties [38] and, consequently, its mechanical function. Both cellular and matrix structures contribute to myocardial physical properties, based on their intrinsic mechanical features, spatial and functional relations with the surrounding structures and tissue geometry, which finally ensues in the adult heart. Thus, when considering heart biomechanical properties, we have to conceptually integrate not only the single contribution of the various cardiac muscle tissue elements but also the complex interactions among them as well as their ability to modify their intrinsic mechanics under different pathophysiological conditions.

Considering the dynamic nature of diastole, it is not surprising that myocardial material properties are a central determinant of diastolic function and dysfunction. Generally 
TABLE 1: Determinants of diastolic function and myocardial stiffness.

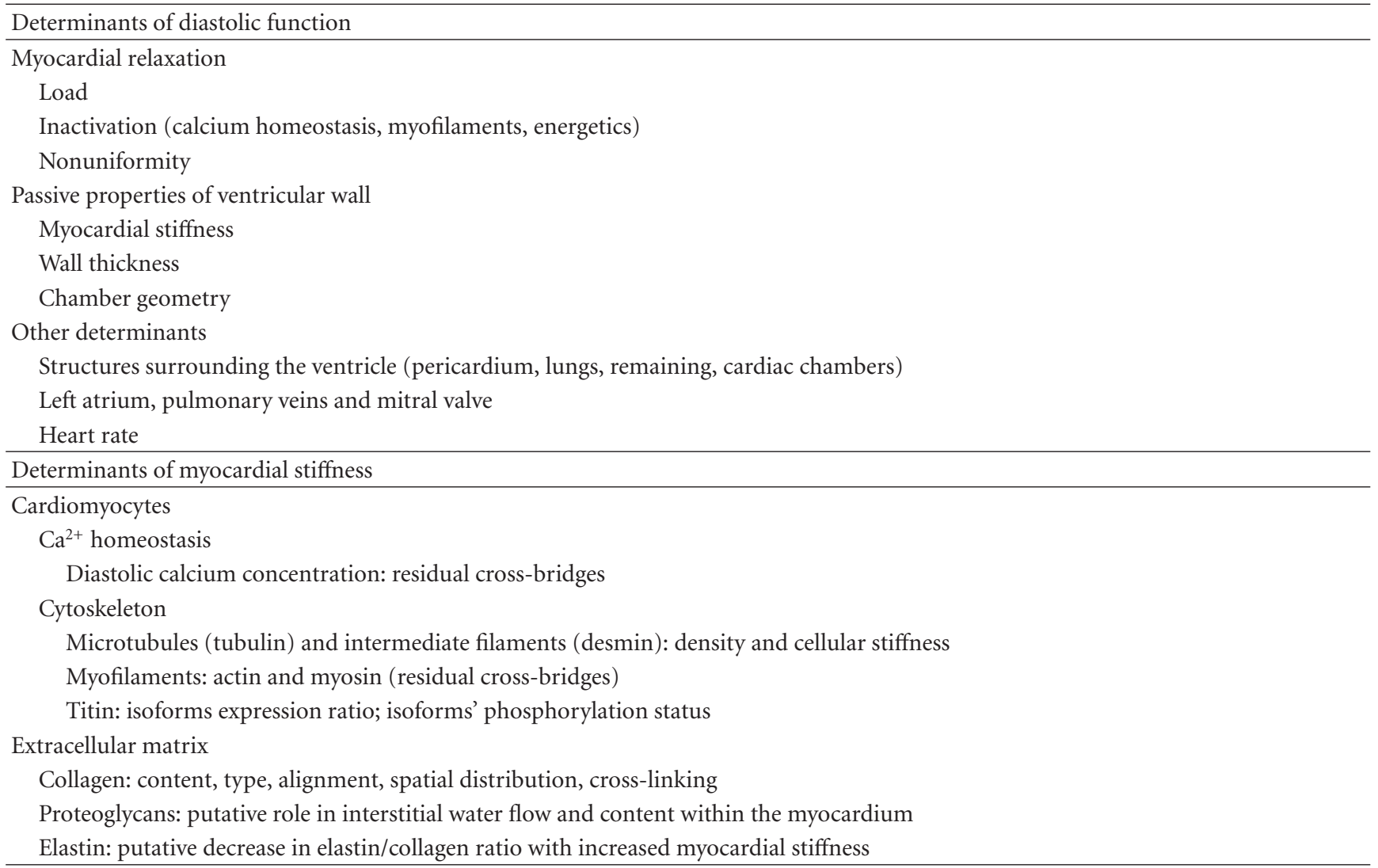

defined as the ability of the ventricle to relax and fill [39], diastolic function depends on active myocardial relaxation and on passive properties. While relaxation is the process whereby the myocardium returns to an unstressed length and force, passive properties mostly influence the extent of muscle relength and end-diastolic pressure-volume relationship (EDPVR). Although active myocardial relaxation is responsible for its motion and blood flow, it is only through the perfect match of both active and passive properties that overall diastolic function is preserved. For conceptual purposes, myocardial passive properties are considered as such because they are assessed after complete muscle relaxation, that is, a mostly energy-consuming and, hence, active process. However, a growing body of evidence clearly shows that myocardial passive properties might as well be actively modulated and depend on cardiac high-energy phosphate metabolism.

The contribution of both active and passive properties to diastolic dysfunction and to the clinical syndrome of HFNEF will be discussed in the next sections.

3.1. Relaxation and Its Physiological Modulators. In the normal heart, myocardial relaxation comprises the major part of ventricular ejection, pressure fall and the initial part of rapid filling [39-42].

At the cellular level, the central players of the relaxation process are the cardiomyocytes, whose homeostatic balance is a major determinant in the maintenance of an appropriate relaxation speed. In this way, any process interfering with cardiomyocyte physiology may delay relaxation long enough and significantly impair LV diastolic filling, especially at faster heart rates.

Conceptually, the determinants of ventricular relaxation (either by disturbing diastolic cardiomyocyte performance or by disrupting the synchrony of their actions) are load, inactivation, and nonuniformity (Table 1) [39].

3.1.1. Load. The effects of load depend on its type (preload versus afterload), magnitude, duration, and timing in the cardiac cycle at which it occurs [40]. For instance, severe or late systolic afterload elevations are particularly effective in delaying relaxation rate, even in healthy hearts [43].

In the clinical setting, marked arterial hypertension is a paradigmatic example of severe afterload imposition on the left ventricle and is a major risk factor for myocardial hypertrophy and failure. As relaxation in failing hearts is especially vulnerable to load, increases in afterload may significantly delay relaxation and result in pulmonary congestion in HF patients [44]. Taking into account that neurohumoral activation takes place both in HFNEF and HFREF, the failing heart in HFNEF is not only faced with a higher afterload (increased peripheral vascular resistance) but also with a higher preload (volume retention), further increasing the odds of hemodynamic decompensation [45]. 
Even if the underlying mechanisms are still unclear, changes in the activity of $\mathrm{Ca}^{2+}$-handling proteins and myofilaments' sensitivity to this ion (e.g., troponin I) are the most likely effectors of the observed slower relaxation upon changes in load [46-49].

3.1.2. Inactivation. Deserving special relief, inactivation refers to the process underlying $\mathrm{Ca}^{2+}$ extrusion from the cytosol and cross-bridge detachment. As abovementioned, its importance stems from the fact that disturbances in this process may represent the ultimate effectors of several pathophysiological conditions leading to impaired relaxation, including load [49].

In the healthy human heart, the pathways involved in $\mathrm{Ca}^{2+}$ extrusion from the cytosol are the phospholambammodulated uptake of this ion by the sarcoplasmic reticulum $\mathrm{Ca}^{2+}$ ATPase (SERCA2a, 70\%), the $\mathrm{Na}^{+} / \mathrm{Ca}^{2+}$-exchanger $(28 \%)$, and, to a lesser extent, the $\mathrm{Ca}^{2+}$-pump and the mitochondrial $\mathrm{Ca}^{2+}$ uniport (2\%) [50]. Equally important is the phosphorylation state of the proteins that regulate SERCA2a activity, such as phospholambam (PLB), calmodulin, and calsequestrin. PLB stands as a classic example of a protein that interacts with and inhibits SERCA2a activity in a reversible manner. Although its relevance in cardiac physiology has been extensively studied over the last years, there is now an emerging field of interest focusing on the structurally-related protein sarcolipin (SLN). Most notably, this protein is absent in the ventricles but is significantly expressed in the atria, which may indicate the existence of chamber-specific mechanisms of SERCA2a function regulation. Nonetheless, overexpression of sarcolipin in mice ventricular myocytes was found to inhibit SERCA2a by direct binding or through stabilization of the interaction between SERCA2a and phospholamban $[51,52]$. As a consequence, the affinity of SERCA2a for calcium was decreased, calcium transients amplitude was lower, and the time of calcium decay and relaxation was significantly extended [53, 54]. Despite the atrial-specific expression of SLN and the higher expression of PLB in the mouse ventricle, SLN expression may not be confined to the atria in other species [54] and possibly in pathological conditions, such as heart failure.

Besides the importance of the aforementioned mechanisms concerning the velocity and extent of $\mathrm{Ca}^{2+}$ reuptake into the SR, it has been proposed that diastolic $\mathrm{Ca}^{2+}$ leakage from the ryanodine receptor (associated with diminished FK 506-binding protein 12.6) may also increase diastolic $\mathrm{Ca}^{2+}$ levels and contribute to a higher thick-thin filament interaction and delayed relaxation [55]. Therefore, the functional integrity of the SR must translate not only in an effective reuptake but also a subsequent diastolic sequestration of $\mathrm{Ca}^{2+}$.

As relaxation is an energy-consuming process, highenergy phosphate metabolism is a centerpiece in several steps involved in this phase of diastole, namely, in $\mathrm{Ca}^{2+}$ dissociation from troponin $\mathrm{C}$, myosin-actin detachment, active sequestration of $\mathrm{Ca}^{2+}$ into the $\mathrm{SR}$, and in several phosphorylation reactions of $\mathrm{Ca}^{2+}$-handling proteins [46]. Indeed, any energetic imbalance leading to an abnormally high ADP concentration or ADP/ATP ratio is associated with slower relaxation rates and increased diastolic stiffness [39]. Besides slower $\mathrm{Ca}^{2+}$ transients' decay, a higher sensitivity of the myofilament proteins to this ion might as well decrease the pace of myocardial relaxation. An important example is cardiac troponin-I (cTnI) which is less phosphorylated in HFREF and therefore shows a higher sensitivity to $\mathrm{Ca}^{2+}$ [56]. As a result, comparable increases in $\mathrm{Ca}^{2+}$ concentration may result in greater myofilaments' activation and enhanced myocardial contractility. However, as corroborated by the recent findings of Yasuda et al., the flip side of the coin may be a delay in myocardial relaxation and a consequent increase in diastolic stiffness. In the latter study, both in vitro and in vivo experiments designed to engineer sarcomeres with a PKA nonphosphorylatable TnI or a PKA phosphomimetic of cardiac TnI indicated an important role of cardiac TnI phosphorylation status in regulating cardiomyocyte relaxation velocity [57].

3.1.3. Nonuniformity. Nonuniformity represents asynchronous changes in ventricular shape during contraction and relaxation, being most frequent in the setting of coronary artery disease and intraventricular conduction disturbances. During isovolumic relaxation, it may be represented by reextension of one ventricular segment accompanied by postsystolic shortening of another, generating an asynchrony in ventricular relengthening and possibly in ventricular pressure fall. Consequently, this may further contribute to diastolic dysfunction [39].

Most, if not all, the risk factors pointed to be associated with HFNEF invariably lead to impaired relaxation, even if some may be especially involved in modifying the myocardial passive properties. However, unraveling the mechanisms through which a single risk factor induces diastolic dysfunction/HFNEF has been a challenging task due to the high prevalence of several comorbidities among HF patients. The latter condition promotes the interaction among the existent risk factors, which may significantly modify the phenotype of the disease, its clinical presentation, and prognosis.

3.2. Ventricular Passive Properties and Their Physiological Modulators. Although active properties of the myocardium are responsible for its motion and blood flow, overall diastolic function is stringently dependent on the maintenance of adequate passive properties as well. Indeed, current data strongly associates changes in myocardial material properties and various forms of heart disease. The passive properties of the ventricular wall depend on myocardial stiffness, wall thickness, and chamber geometry (Table 1) and are evaluated by observing the position and shape of the EDPVR [39]. This is an exponential curve which is derived by plotting the lower right corner of multiple pressure-volume (PV) loops at various preloads. Considering the three-parameter monoexponential formula $\left(P=P_{0}+A^{*} e^{k c^{*} V}\right)$, the EDPVR is dependent on ventricular distensibility (represented by changes in vertical position, i.e., in the value of the pressure intercept $P_{0}$ ), ventricular size (represented by changes in horizontal position, that is, in constant $A$ ), and ventricular 


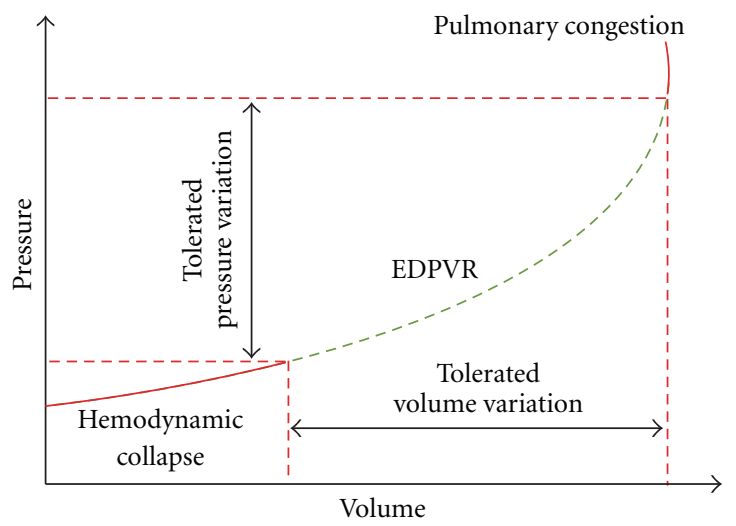

(a)

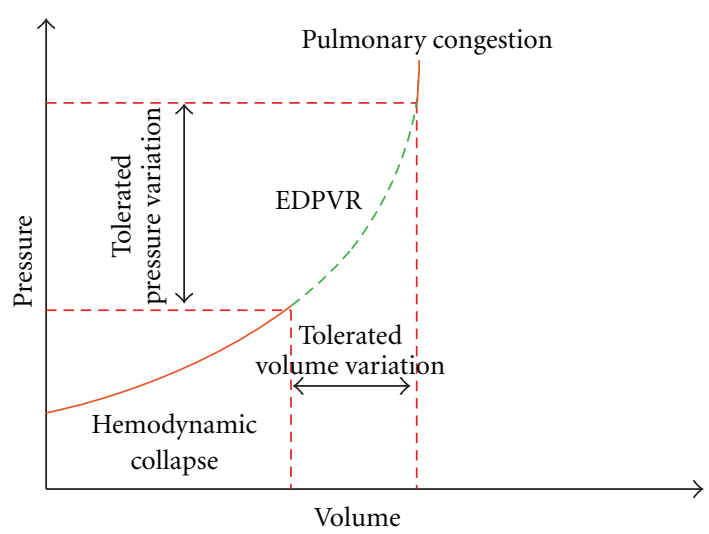

(b)

FIGURE 1: Schematic representation of the end-diastolic pressure-volume relationship (EDPVR) in the absence (a) or presence (b) of diastolic dysfunction. The dashed segment of the EDPVR represents the pressure-volume interval in which an individual remains hemodynamically stable. In (b), the steeper EDPVR narrows the ventricular pressure-volume interval and increases the individual's susceptibility to hemodynamic decompensation.

compliance (represented by changes in the slope of the curve, i.e., in the modulus of chamber stiffness, $K c$ ).

Due to the concentric hypertrophy and stiffer myocardial wall commonly associated with HFNEF, the hallmarks of this condition are decreased distensibility, decreased compliance, and smaller cavity size. As a consequence, the ventricular wall displays stronger elastic forces resisting expansion during diastolic filling, which is translated into an upward and leftward shift of the EDPVR, that is, into a steeper relation. Indeed, changes in its slope deserve special emphasis as it reflects the patient's vulnerability to hemodynamic decompensation, regardless of the HF phenotype (HFNEF or HFREF) and ventricular cavity size [45].

As depicted in Figure 1, the slope of the curve at operating conditions is a valuable surrogate of these patients' clinical susceptibility: the steeper the curve, the smaller the volume variation capable of markedly increasing ventricular filling pressures and inducing pulmonary congestion.

However, we must bear in mind that the EDPVR is not solely dependent on intrinsic passive properties of the ventricular wall but also on extrinsic factors (e.g., pericardium, lungs) $[39,46]$ as well as conditions leading to incomplete myocardial relaxation, such as severe tachycardia in HF patients (Table 1). The latter situation is especially important because their hearts may exhibit a flat or even negative relaxation velocity-versus-heart rate relationship [58]. In this way, when heart rate increases, relaxation rate does not increase or even decreases, leading to a stiffer ventricle in late diastole and hence higher filling pressures.

3.2.1. Myocardial Stiffness. Considering the importance of myocardial material properties in determining ventricular compliance and clinical severity of HFNEF, their study became an attractive field in HF research. In further sections, there will be emphasized the relative contribution of cardiomyocytes and extracelular matrix to overall myocardial stiffness, both in physiological and pathological conditions (Table 1).

\section{Cardiomyocytes and Myocardial Material Properties}

Several studies have shown that changes in myocardial material properties can be caused by mechanisms intrinsic to the cardiomyocytes themselves [38,59-68]. These include mechanisms that may alter (i) the relative content, (ii) the isoforms expression, (iii) posttranslational modifications and/or (iv) active interactions among the cytoskeletal structures of cardiac cells, thus affecting the overall resistance to changes in shape.

The cardiomyocyte cytoskeleton is composed of microtubules (tubulin), intermediate filaments (desmin), microfilaments (actin), and endosarcomeric proteins, of which titin has lately received particular attention.

4.1. Microtubules and Intermediate Filaments. At operating sarcomere lengths $(1.9-2.2 \mu \mathrm{m})$, microtubules and intermediate filaments were found to contribute less than $10 \%$ to passive tension [67]. However, in the setting of right-ventricular pressure overload hypertrophy (RVPOH) and at physiological rates of muscle length variation, there is a higher resistance to changes in cardiomyocyte shape, a result that is significantly attenuated when microtubules are chemically or physically depolymerized. Thus, although microtubules may not significantly contribute to myocardial stiffness both in healthy states and in slowly stretched muscles, their increased density may play a role under pressure-overloaded conditions and at physiological rates of contraction and relaxation $[60,67]$. In these conditions, microtubules are mainly responsible for an increase in cardiomyocyte resistance when rapidly stretched.

4.2. Myofilaments. Since the interaction between actin and myosin was shown to occur even at low diastolic calcium levels, it was hypothesized that the establishment of residual 
diastolic cross-bridges might as well mediate myocardial passive stiffness [46]. As described for microtubules, Zile and colleagues also showed that, in pressure-overloaded conditions, there is a correlation between increased myocardial stiffness, higher intracellular calcium concentration, and increased number of cross-bridge interactions [38]. Therefore, changes in calcium transients or myofilament's calcium-sensitivity may increase myocardial stiffness $[46,69]$ even if these are most commonly regarded as determinants of myocardial relaxation rate. The latter findings mean that beyond the biophysical properties of structural cytoskeletal proteins, cardiomyocyte stiffness may also be under active control, thus evoking the concept of active muscle tone [46].

4.3. Titin. Over the last decade, the contribution of the endosarcomeric protein titin in the modulation of myocardial stiffness has been elucidated. However, its relevance in cardiac function goes beyond the scaffold and passive force generating properties, being an important regulator of systolic function $[70,71]$, a mechanotransducer in cardiomyocytes [72], and a central player in cardiac development, growth, and hypertrophy [73-75].

4.3.1. Titin Isoforms and Myocardial Stiffness. Titin spans the half-sarcomere from the Z-disk to M-line and its ability to generate passive tension is associated with extensible segments in the I-band of sarcomeres, comprised of serially linked but distinct domains: the proline-glutamate-valinelysine (PEVK) element, immunoglobulin-(Ig-)like domains and the segment with unique aminoacid sequences called $\mathrm{N} 2 \mathrm{~B}$, N2A, or N2BA (which contains both N2B and $\mathrm{N} 2 \mathrm{~A})[46,59,61,63,76]$. It is expressed in the heart as two different isoforms, the smaller and stiffer N2B, and the larger and more compliant N2BA. While the former expresses only the cardiac specific $\mathrm{N} 2 \mathrm{~B}$ domain, the latter expresses both $\mathrm{N} 2 \mathrm{~B}$ and $\mathrm{N} 2 \mathrm{~A}$ as well as additional PEVK and Ig domains. Upon application of a longitudinal force, stretch of cardiomyocytes within physiological sarcomere length ranges is accompanied by unfolding of the PEVK, Ig domains, and unique sequences N2A and/or N2B, resulting in the generation of passive force. Upon release, these spring elements tend to refold and achieve a state of lower contour length and higher entropy. Given the presence of additional segments in the N2BA titin isoform, it can accommodate longer variations in length for the same levels of passive force generated, being therefore more compliant than N2B isoform. However, because these isoforms are coexpressed in the same half sarcomere in a ratio that varies among species and in different locations within the heart, titinbased cardiomyocyte stiffness is usually anywhere in-between of that generated by N2B- or N2BA-purely expressing cells. Accordingly, the predominant expression of N2B in rodents $[46,69]$ accounts for a high cardiomyocyte stiffness, while the more abundant expression of N2BA in large mammals (pig, cow, human) accounts for a comparably lower passive stiffness. However, and regardless of the animal species, myocardial stiffness undergoes significant changes during physiological and pathological conditions, which are partially accounted by shifts in titin isoforms expression ratio as well as titin posttranslational modifications. The earliest and probably the most robust shift occurs during the perinatal period. In the fetal myocardium of mice, rats, and pigs, titin is predominantly expressed as a compliant N2BA titin isoform (fetal cardiac titin), which is characterized by the presence of additional Ig and PEVK segments in its extensible region than the adult N2BA titin [77]. Around birth, this fetal isoform is downregulated and titin transcripts are translated into a shorter, less extensible N2BA protein, together with the onset $\mathrm{N} 2 \mathrm{~B}$ titin isoform expression. With a time course that varies among species, N2B becomes the predominant isoform in the adult heart, including humans. As a consequence, the highly compliant fetal myocardium becomes progressively stiffer and better adapted to higher ventricular end-diastolic pressures that accompany postnatal cardiac growth. Even in adulthood, titin isoforms expression ratio is highly dynamic and contributes to changes in myocardial stiffness upon pathological insults. However, the direction of isoform shift is not consistent among studies and the mechanisms that may lead to one type of switch over another in HF remain unclear [46, 59]. A plausible explanation for these discrepancies relies on the different experimental/clinical contexts where the shift in titin isoforms expression was studied, mostly likely on differences in the nature, severity, and duration of the disease. As depicted in Figure 2, the development of HF or HF-associated pathological conditions is accompanied by an increase in myocardial stiffness (Figure 2(a)), where remodeling of the ECM invariably leads to a higher collagenbased stiffness (Figure 2(b)). However, while in 2 canine models of pacing-induced dilated cardiomyopathy (DCM) $[78,79]$ the increase in myocardial stiffness with HF was accompanied by a higher expression of the stiff N2B titin isoform (Figure 2(c)), the opposite was observed in HF human hearts, either with normal [80] or reduced ejection fraction [81-83]. (Figure 2(d)). In these studies, the net increase in myocardial stiffness was accounted by a higher collagen-based stiffness and/or changes titin isoforms phosphorylation ratio. The observed increase in $\mathrm{N} 2 \mathrm{BA} / \mathrm{N} 2 \mathrm{~B}$ expression ratio has been rather interpreted as a compensatory mechanism in which the presence of more compliant titin isoforms counteracts the progressive increase in myocardial stiffness and diastolic dysfunction. Similar findings in titin isoforms shift have been recently reported in long-term hypothyroidism, a condition commonly associated with diastolic dysfunction [84]. Of note, Figure 2 does not take into consideration the relative proportions of $\mathrm{N} 2 \mathrm{~B}$ and N2BA titin isoforms but rather the main direction of their shift, as reported in several studies.

\subsubsection{Titin Posttranslational Modifications and Myocardial} Stiffness. Besides the molecular structure and relative expression ratio, posttranslational modifications of titin isoforms (through either phosphorylation or calcium binding effects) may significantly impact myocardial stiffness. N2B titin's segment was shown to be the target for protein kinase A 
Fetal/

neonatal

(a)

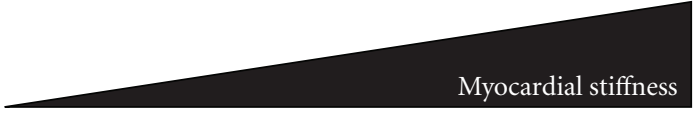

(b)

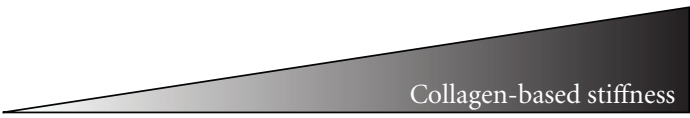

(c)

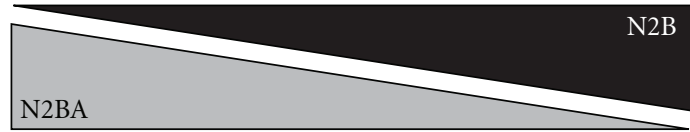

(d)

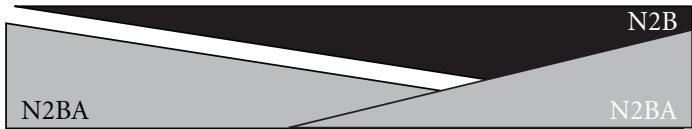

(e)

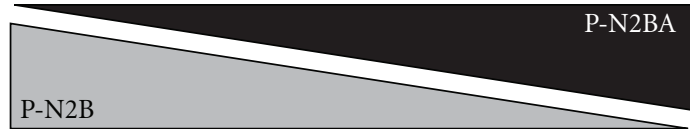

Figure 2: Progressive increase in myocardial (a) and collagen-based stiffness (b) during physiologic cardiac growth as well as HF or HF-associated pathological conditions. The increase in myocardial stiffness can be paralleled by a concordant increase in the expression of the stiff titin isoform N2B (c) or by a compensatory increase in the expression of the compliant titin isoform N2BA (d). Relative hypophosphorylation of the stiff N2B titin also accounts for an increase in myocardial stiffness in HF, especially in HFNEF (e) (PN2B: phosphorylated N2B; P-N2BA: phosphorylated N2BA).

(PKA) and protein kinase G (PKG) phosphorylation $[61,76$, 85-87], which possibly destabilizes its structure and induces an increase in N2B segment length, thus reducing cardiomyocyte passive tension. Because N2B element is included in all cardiac titin isoforms, its extension is predicted to reduce passive stiffness of both N2B and N2BA titins. However, the magnitude of the phosphorylation-induced decrease in passive stiffness is isoform dependent, because the shorter N2B titin makes its increase in length to have a greater impact on its fractional extension. Accordingly, a relative deficit in N2B titin phosphorylation status was found to significantly raise cardiomyocyte stiffness in failing human myocardium (Figure 2(e)), despite the compensatory increase in N2BA titin isoform expression and phosphorylation. Interestingly, patients with HFNEF had not only more N2B titin protein (i.e., stiffer) but also less phosphorylated N2B titin (i.e., stiffer) than HFREF patients, explaining both the significantly higher stiffness and the more pronounced decay in of passive tension upon to PKA-mediated phosphorylation [80].

Titin phosphorylation by PKA and PKG clearly supports the effects of $\beta$-adrenergic signaling and nitric oxide in reducing cardiomyocyte stiffness, respectively $[86,87]$. However, the association between titin phosphorylation by Protein Kinase C (PKC) and the reported effects of several agonists of $\mathrm{Gq}$ protein-coupled receptors is not straightforward. As recently published, PKC phosphorylates titin's
PEVK segment and leads to an increase in cardiomyocyte stiffness $[88,89]$ suggesting that the adaptation of myocardial stiffness upon adrenergic stimulation may be finely regulated by a balance between alpha and beta adrenergic receptors' activation. However, acute activation of other Gq protein coupled receptors (AT1, $\mathrm{ET}_{A}$, among others) has been associated with a decrease in myocardial stiffness, an effect that was blunted when PKC was specifically inhibited [90, 91]. Whether a broader effect of PKC on the phosphorylation status of cardiac myofilaments, the isoform of PKC or the simultaneous activation of other pathways may have accounted for these results warrants further assessment.

Besides titin extention, diastolic stiffness may be also produced by titin-calcium interactions in an isoform dependent manner. Calcium binding directly increases the passive stiffness of N2BA expressing myocardium by changes in its structure but has no effect on exclusively N2B expressing myocardium. In the latter case, calcium exerts an indirect effect by promoting titin-actin interactions, which may serve to retard thin filament sliding and contribute to myocyte passive stiffness [59].

\section{ExtraCellular Matrix and Myocardial Material Properties}

Besides cardiomyocytes, the ECM is another major determinant of the myocardial material properties [92]. Among the proteins within the ECM, fibrillar proteins such as collagen and elastin, proteoglycans, and the basement membrane proteins each may play a role in determining the constitutive properties of the myocardium [93, 94]. However, collagen molecules have been hypothesized to be the most important component within the ECM contributing to myocardial stiffness and HFNEF [46], as it is a relatively stiff material with high tensile strength. However, its influence on the stress-strain relation of the myocardium depends on many factors including its concentration, fibril and fiber diameter, degree of crosslinking, spatial alignment, and collagen types. In physiological conditions and at sarcomere lengths less than $2.2 \mu \mathrm{m}$, the combined passive fiber stiffness of the myocardium has been predominantly attributed to intracellular structures, notably titin. However, at longer sarcomere lengths, parallel collagen fibers bear an increasing fraction of the axial stress as collagen perimysial fibers untwist and straighten [94]. Interestingly enough, the increase in myocardial stiffness across different species and heart chambers is associated not only to a higher expression of the stiff N2B titin isoform but also by a parallel increase in collagen content, which suggests coordination between collagen synthesis and titin isoform expression. This might preserve the relative stiffness contributions of titin (lower sarcomere lengths) and collagen (higher sarcomere lengths) among species. However, this relationship may not be preserved in pathological states [46] such as arterial hypertension, diabetes $[95,96]$, or myocardial infarction [97, 98], because of their role in disrupting the balance between collagen biosynthesis, degradation, and posttranslational processing [46]. 


\section{Neurohumoral Modulation of Myocardial Mechanical Properties}

Besides mechanical inputs, growing evidence points to neurohumoral agents such as nitric oxide (NO) [99-101], angiotensin II [90, 102], endothelin-1 [91, 103, 104], urotensin-II $[105,106]$, and adrenomedullin [107] to acutely alter myocardial mechanical properties as well [108].

In isolated cardiomyocytes, $\mathrm{NO}$ was shown to shift the stress-strain relation down and to the right. Accordingly, intracoronary infusion of the exogenous NO donor, sodium nitroprusside, resulted in a similar displacement of the enddiastolic pressure-volume relation, both in normal and in hypertrophied human hearts as a result PKG-mediated phosphorylation of myofilaments [101]. In further experiments, substance $\mathrm{P}$ was shown to promote the release of endogenous $\mathrm{NO}$, decreasing ventricular stiffness in patients with dilated cardiomyopathy $[39,102]$. Although some previous reports [109] stated that higher coronary perfusion pressures might cause an upward displacement of EDPVR by increasing myocardial vascular engorgement, the latter results also suggest that a simultaneous increase in endothelial shearstress may enhance the release of endogenous $\mathrm{NO}$ and attenuate the aforementioned effect. As titin is currently known to be a target of PKG phosphorylation, an increase in titin distensibility might have as well contributed to the observed effects [87].

Chronic activation of the renin-angiotensin system is also a well-recognized mechanism that leads to increased myocardial stiffness by promoting structural remodeling in both cardiomyocytes and ECM. However, acute activation of this system was shown to decrease myocardial and ventricular chamber stiffness in a time frame that was too short to alter the ECM [90]. Therefore, its effects on myocardial tissue must be caused by direct action on the cardiomyocytes to alter one or more determinants of its mechanical properties. Interestingly, a similar finding was also ascribed to endothelin-1 in isolated papillary muscles [91]. As angiotensin II and endothelin-1 exerted their effects through the activation of Gq Protein-Coupled Receptors which are established activators of PKC signaling, further studies are necessary to reconcile these observations with the recently described PKC-mediated increase in titin and cardiomyocyte stiffness.

\section{Conclusion}

The maintenance of normal diastolic performance is only possible through the appropriate expression of cellular and matrix phenotypes, which in turn are dependent on the perfect match between the myocardial inputs and its biological responses. Myocardial tissue is regarded as composite material whose properties depend on each of its specific constituent elements and the dynamic interplay between their structure and function. In addition, the biomechanical sensing features of the myocardium allow it to alter its structural and functional phenotypes when subjected to physiological inputs, thus achieving a new biological equilibrium that preserves overall ventricular function. In pathological settings, however, such equilibrium can be easily disrupted, with both cardiomyocytes and ECM exhibiting changes that may impair ventricular relaxation and chamber stiffness. The growing knowledge about the causal mechanisms of abnormal myocardial properties will possibly allow us to diagnose earlier and choose the best therapeutic strategies in patients with HF in general and HFNEF in particular.

\section{References}

[1] J. Lammerding, R. D. Kamm, and R. T. Lee, "Mechanotransduction in cardiac myocytes," Annals of the New York Academy of Sciences, vol. 1015, pp. 53-70, 2004.

[2] M. A. Sussman, A. McCulloch, and T. K. Borg, "Dance band on the Titanic: biomechanical signaling in cardiac hypertrophy," Circulation Research, vol. 91, no. 10, pp. 888898, 2002.

[3] A. W. Orr, B. P. Helmke, B. R. Blackman, and M. A. Schwartz, "Mechanisms of mechanotransduction," Developmental Cell, vol. 10, no. 1, pp. 11-20, 2006.

[4] B. Swynghedauw, "Phenotypic plasticity of adult myocardium: molecular mechanisms," Journal of Experimental Biology, vol. 209, no. 12, pp. 2320-2327, 2006.

[5] D. D. Bonnema, C. S. Webb, W. R. Pennington, et al., "Effects of age on plasma matrix metalloproteinases (MMPs) and tissue inhibitor of metalloproteinases (TIMPs)," Journal of Cardiac Failure, vol. 13, no. 7, pp. 530-540, 2007.

[6] B. P. Shapiro, C. S. P. Lam, J. B. Patel, et al., "Acute and chronic ventricular-arterial coupling in systole and diastole: insights from an elderly hypertensive model," Hypertension, vol. 50, no. 3, pp. 503-511, 2007.

[7] H. Masugata, S. Senda, F. Goda, et al., "Left ventricular diastolic dysfunction in normotensive diabetic patients in various age strata," Diabetes Research and Clinical Practice, vol. 79, no. 1, pp. 91-96, 2008.

[8] L. van Heerebeek, N. Hamdani, M. L. Handoko, et al., "Diastolic stiffness of the failing diabetic heart: importance of fibrosis, advanced glycation end products, and myocyte resting tension," Circulation, vol. 117, no. 1, pp. 43-51, 2008.

[9] C. Y. Wong, T. O’Moore-Sullivan, R. Leano, N. Byrne, E. Beller, and T. H. Marwick, "Alterations of left ventricular myocardial characteristics associated with obesity," Circulation, vol. 110, no. 19, pp. 3081-3087, 2004.

[10] B. D. Powell, M. M. Redfield, K. A. Bybee, W. K. Freeman, and C. S. Rihal, "Association of obesity with left ventricular remodeling and diastolic dysfunction in patients without coronary artery disease," American Journal of Cardiology, vol. 98, no. 1, pp. 116-120, 2006.

[11] J. F. Carroll, R. L. Summers, D. J. Dzielak, K. Cockrell, J.-P. Montani, and H. L. Mizelle, "Diastolic compliance is reduced in obese rabbits," Hypertension, vol. 33, no. 3, pp. 811-815, 1999.

[12] J. M. McGavock, R. G. Victor, R. H. Unger, and L. S. Szczepaniak, "Adiposity of the heart, revisited," Annals of Internal Medicine, vol. 144, no. 7, pp. 517-524, 2006.

[13] M. Pascual, D. A. Pascual, F. Soria, et al., "Effects of isolated obesity on systolic and diastolic left ventricular function," Heart, vol. 89, no. 10, pp. 1152-1156, 2003. 
[14] J. F. Carroll, D. S. Braden, K. Cockrell, and H. L. Mizelle, "Obese hypertensive rabbits develop concentric and eccentric hypertrophy and diastolic filling abnormalities," American Journal of Hypertension, vol. 10, no. 2, pp. 230-233, 1997.

[15] L. R. Peterson, A. D. Waggoner, K. B. Schechtman, et al., "Alterations in left ventricular structure and function in young healthy obese women: assessment by echocardiography and tissue Doppler imaging," Journal of the American College of Cardiology, vol. 43, no. 8, pp. 1399-1404, 2004.

[16] V. Regitz-Zagrosek, S. Brokat, and C. Tschope, "Role of gender in heart failure with normal left ventricular ejection fraction," Progress in Cardiovascular Diseases, vol. 49, no. 4, pp. 241-251, 2007.

[17] F. A. Masoudi, E. P. Havranek, G. Smith, et al., "Gender, age, and heart failure with preserved left ventricular systolic function," Journal of the American College of Cardiology, vol. 41, no. 2, pp. 217-223, 2003.

[18] P. R. Mitoff, A. Al-Hesayen, E. Azevedo, G. E. Newton, and S. Mak, "Sex differences in basal hemodynamics and left ventricular function in humans with and without heart failure," American Heart Journal, vol. 154, no. 3, pp. 575-580, 2007.

[19] M. M. Redfield, S. J. Jacobsen, B. A. Borlaug, R. J. Rodeheffer, and D. A. Kass, "Age- and gender-related ventricular-vascular stiffening: a community-based study," Circulation, vol. 112, no. 15, pp. 2254-2262, 2005.

[20] Y. Izumi, K. Matsumoto, Y. Ozawa, et al., "Effect of age at menopause on blood pressure in postmenopausal women," American Journal of Hypertension, vol. 20, no. 10, pp. 10451050, 2007.

[21] C. S. P. Lam, V. L. Roger, R. J. Rodeheffer, et al., "Cardiac structure and ventricular-vascular function in persons with heart failure and preserved ejection fraction from Olmsted County, Minnesota," Circulation, vol. 115, no. 15, pp. 19821990, 2007.

[22] W. J. Paulus, C. Tschöpe, J. E. Sanderson, et al., "How to diagnose diastolic heart failure: a consensus statement on the diagnosis of heart failure with normal left ventricular ejection fraction by the Heart Failure and Echocardiography Associations of the European Society of Cardiology," European Heart Journal, vol. 28, no. 20, pp. 2539-2550, 2007.

[23] W. J. Paulus and J. J. M. van Ballegoij, "Treatment of heart failure with normal ejection fraction. An inconvenient truth!," Journal of the American College of Cardiology, vol. 55, no. 6, pp. 526-537, 2010.

[24] S. S. Najjar, "Heart failure with preserved ejection fraction. Failure to preserve, failure of reserve, and failure on the compliance curve," Journal of the American College of Cardiology, vol. 54, no. 5, pp. 419-421, 2009.

[25] L. van Heerebeek, A. Borbély, H. W. M. Niessen, et al., "Myocardial structure and function differ in systolic and diastolic heart failure," Circulation, vol. 113, no. 16, pp. 19661973, 2006.

[26] M. Klapholz, M. Maurer, A. M. Lowe, et al., "Hospitalization for heart failure in the presence of a normal left ventricular ejection fraction: results of the New York heart failure registry," Journal of the American College of Cardiology, vol. 43, no. 8, pp. 1432-1438, 2004.

[27] D. S. Lee, P. Gona, R. S. Vasan, et al., "Relation of disease pathogenesis and risk factors to heart failure with preserved or reduced ejection fraction: insights from the framingham heart study of the national heart, lung, and blood institute," Circulation, vol. 119, no. 24, pp. 3070-3077, 2009.
[28] E. O’Meara, T. Clayton, M. B. McEntegart, et al., "Sex differences in clinical characteristics and prognosis in a broad spectrum of patients with heart failure-results of the Candesartan in Heart failure: assessment of reduction in mortality and morbidity (CHARM) program," Circulation, vol. 115, no. 24, pp. 3111-3120, 2007.

[29] V. M. Vizgirda, G. M. Wahler, K. L. Sondgeroth, M. T. Ziolo, and D. W. Schwertz, "Mechanisms of sex differences in rat cardiac myocyte response to $\beta$-adrenergic stimulation," American Journal of Physiology, vol. 282, no. 1, pp. H256H263, 2002.

[30] R. Dash, K. F. Frank, A. N. Carr, C. S. Moravec, and E. G. Kranias, "Gender influences on sarcoplasmic reticulum $\mathrm{Ca}^{2+}$-handling in failing human myocardium," Journal of Molecular and Cellular Cardiology, vol. 33, no. 7, pp. 13451353, 2001.

[31] D. W. Schwertz, V. Vizgirda, R. J. Solaro, M. R. Piano, and C. Ryjewski, "Sexual dimorphism in rat left atrial function and response to adrenergic stimulation," Molecular and Cellular Biochemistry, vol. 200, no. 1-2, pp. 143-153, 1999.

[32] S. Jovanović, A. Jovanović, W. K. Shen, and A. Terzic, "Low concentrations of $17 \beta$-estradiol protect single cardiac cells against metabolic stress-induced $\mathrm{Ca}^{2+}$ loading," Journal of the American College of Cardiology, vol. 36, no. 3, pp. 948-952, 2000.

[33] M. van Eickels, C. Grohé, J. P. M. Cleutjens, B. J. Janssen, H. J. J. Wellens, and P. A. Doevendans, "17 $\beta$-estradiol attenuates the development of pressure-overload hypertrophy," Circulation, vol. 104, no. 12, pp. 1419-1423, 2001.

[34] Y. Xu, I. A. Arenas, S. J. Armstrong, and S. T. Davidge, "Estrogen modulation of left ventricular remodeling in the aged heart," Cardiovascular Research, vol. 57, no. 2, pp. 388394, 2003.

[35] B. Kuch, M. Muscholl, A. Luchner, et al., "Gender specific differences in left ventricular adaptation to obesity and hypertension," Journal of Human Hypertension, vol. 12, no. 10, pp. 685-691, 1998.

[36] V. Regitz-Zagrosek and E. Lehmkuhl, "Heart failure and its treatment in women: role of hypertension, diabetes, and estrogen," Herz, vol. 30, no. 5, pp. 356-367, 2005.

[37] W. B. Kannel, M. Hjortland, and W. P. Castelli, "Role of diabetes in congestive heart failure: the Framingham study," American Journal of Cardiology, vol. 34, no. 1, pp. 29-34, 1974.

[38] T. S. Harris, C. F. Baicu, C. H. Conrad, et al., "Constitutive properties of hypertrophied myocardium: cellular contribution to changes in myocardial stiffness," American Journal of Physiology, vol. 282, no. 6, pp. H2173-H2182, 2002.

[39] A. F. Leite-Moreira, "Current perspectives in diastolic dysfunction and diastolic heart failure," Heart, vol. 92, no. 5, pp. 712-718, 2006.

[40] T. C. Gillebert, A. F. Leite-Moreira, and S. G. De Hert, "Load dependent diastolic dysfunction in heart failure," Heart Failure Reviews, vol. 5, no. 4, pp. 345-355, 2000.

[41] T. C. Gillebert, A. F. Leite-Moreira, and S. G. De Hert, “The hemodynamic manifestation of normal myocardial relaxation. A framework for experimental and clinical evaluation," Acta Cardiologica, vol. 52, no. 3, pp. 223-246, 1997.

[42] T. C. Gillebert, A. F. Leite-Moreira, and S. G. De Hert, "Relaxation-systolic pressure relation: a load-independent assessment of left ventricular contractility," Circulation, vol. 95, no. 3, pp. 745-752, 1997. 
[43] A. F. Leite-Moreira and J. Correia-Pinto, "Load as an acute determinant of end-diastolic pressure-volume relation," American Journal of Physiology, vol. 280, no. 1, pp. H51-H59, 2001.

[44] S. K. Gandhi, J. C. Powers, A.-M. Nomeir, et al., "The pathogenesis of acute pulmonary edema associated with hypertension," The New England Journal of Medicine, vol. 344, no. 1, pp. 17-22, 2001.

[45] B. A. Borlaug and D. A. Kass, "Mechanisms of diastolic dysfunction in heart failure," Trends in Cardiovascular Medicine, vol. 16, no. 8, pp. 273-279, 2006.

[46] D. A. Kass, J. G. F. Bronzwaer, and W. J. Paulus, "What mechanisms underlie diastolic dysfunction in heart failure?" Circulation Research, vol. 94, no. 12, pp. 1533-1542, 2004.

[47] A. F. Leite-Moreira, J. Correia-Pinto, and T. C. Gillebert, "Load dependence of left ventricular contraction and relaxation. Effects of caffeine," Basic Research in Cardiology, vol. 94, no. 4, pp. 284-293, 1999.

[48] S. F. J. Langer and H. D. Schmidt, "Different left ventricular relaxation parameters in isolated working rat and guinea pig hearts: influence of preload, afterload, temperature, and isoprenaline," International Journal of Cardiac Imaging, vol. 14, no. 4, pp. 229-240, 1998.

[49] A. F. Leite-Moreira, J. Correia-Pinto, and T. C. Gillebert, "Afterload induced changes in myocardial relaxation: a mechanism for diastolic dysfunction," Cardiovascular Research, vol. 43, no. 2, pp. 344-353, 1999.

[50] D. M. Bers, "Cardiac excitation-contraction coupling," Nature, vol. 415, no. 6868, pp. 198-205, 2002.

[51] A. R. Tupling, M. Asahi, and D. H. MacLennan, "Sarcolipin overexpression in rat slow twitch muscle inhibits sarcoplasmic reticulum $\mathrm{Ca}^{2+}$ uptake and impairs contractile function," Journal of Biological Chemistry, vol. 277, no. 47, pp. 44740 44746, 2002.

[52] M. Asahi, Y. Sugita, K. Kurzydlowski, et al., "Sarcolipin regulates sarco(endo)plasmic reticulum $\mathrm{Ca}^{2+}$-ATPase (SERCA) by binding to transmembrane helices alone or in association with phospholamban," Proceedings of the National Academy of Sciences of the United States of America, vol. 100, no. 9, pp. 5040-5045, 2003.

[53] G. J. Babu, P. Bhupathy, N. N. Petrashevskaya, et al., "Targeted overexpression of sarcolipin in the mouse heart decreases sarcoplasmic reticulum calcium transport and cardiac contractility," Journal of Biological Chemistry, vol. 281, no. 7, pp. 3972-3979, 2006.

[54] M. Asahi, K. Otsu, H. Nakayama, et al., "Cardiac-specific overexpression of sarcolipin inhibits sarco(endo)plasmic reticulum $\mathrm{Ca}^{2+}$ ATPase (SERCA2a) activity and impairs cardiac function in mice," Proceedings of the National Academy of Sciences of the United States of America, vol. 101, no. 25, pp. 9199-9204, 2004.

[55] S. O. Marx, S. Reiken, Y. Hisamatsu, et al., "PKA phosphorylation dissociates FKBP12.6 from the calcium release channel (ryanodine receptor): defective regulation in failing hearts," Cell, vol. 101, no. 4, pp. 365-376, 2000.

[56] J. van der Velden, Z. Papp, R. Zaremba, et al., "Increased $\mathrm{Ca}^{2+}$-sensitivity of the contractile apparatus in end-stage human heart failure results from altered phosphorylation of contractile proteins," Cardiovascular Research, vol. 57, no. 1, pp. 37-47, 2003.

[57] S.-I. Yasuda, P. Coutu, S. Sadayappan, J. Robbins, and J. M. Metzger, "Cardiac transgenic and gene transfer strategies converge to support an important role for troponin I in regulating relaxation in cardiac myocytes," Circulation Research, vol. 101, no. 4, pp. 377-386, 2007.

[58] M. R. Zile and D. L. Brutsaert, "New concepts in diastolic dysfunction and diastolic heart failure: part II. Causal mechanisms and treatment," Circulation, vol. 105, no. 12, pp. 1503-1508, 2002.

[59] M. M. LeWinter, Y. Wu, S. Labeit, and H. Granzier, "Cardiac titin: structure, functions and role in disease," Clinica Chimica Acta, vol. 375, no. 1-2, pp. 1-9, 2007.

[60] H. Shintani and S. A. Glantz, "The left ventricular diastolic pressure-volume relation, relaxation and filling," in Left Ventricular Diastolic Dysfunction and Heart Failure, W. H. Gaash and M. M. LeWinter, Eds., pp. 3-24, Lea \& Febiger, Philadelphia, Pa, USA, 1994.

[61] H. L. Granzier and S. Labeit, "The giant protein titin: a major player in myocardial mechanics, signaling and disease," Circulation Research, vol. 94, no. 3, pp. 284-295, 2004.

[62] A. J. Brady, "Length dependence of passive stiffness in single cardiac myocytes," American Journal of Physiology, vol. 260, no. 4, pp. H1062-H1071, 1991.

[63] M. Helmes, C. C. Lim, R. Liao, A. Bharti, L. Cui, and D. B. Sawyer, "Titin determines the Frank-Starling relation in early diastole," Journal of General Physiology, vol. 121, no. 2, pp. 97-110, 2003.

[64] K. Trombitas, A. Redkar, T. Centner, Y. Wu, S. Labeit, and H. Granzier, "Extensibility of isoforms of cardiac titin: variation in contour length of molecular subsegments provides a basis for cellular passive stiffness diversity," Biophysical Journal, vol. 79, no. 6, pp. 3226-3234, 2000.

[65] S. Nishimura, S.-I. Yasuda, M. Katoh, et al., "Single cell mechanics of rat cardiomyocytes under isometric, unloaded, and physiologically loaded conditions," American Journal of Physiology, vol. 287, no. 1, pp. H196-H202, 2004.

[66] A. Borbély, J. van der Velden, Z. Papp, et al., "Cardiomyocyte stiffness in diastolic heart failure," Circulation, vol. 111, no. 6, pp. 774-781, 2005.

[67] H. L. Granzier and T. C. Irving, "Passive tension in cardiac muscle: contribution of collagen, titin, microtubules, and intermediate filaments," Biophysical Journal, vol. 68, no. 3, pp. 1027-1044, 1995.

[68] M. R. Zile, K. Richardson, M. K. Cowles, et al., "Constitutive properties of adult mammalian cardiac muscle cells," Circulation, vol. 98, no. 6, pp. 567-579, 1998.

[69] O. Cazorla, A. Freiburg, M. Helmes, et al., "Differential expression of cardiac titin isoforms and modulation of cellular stiffness," Circulation Research, vol. 86, no. 1, pp. 5967, 2000.

[70] O. Cazorla, Y. Wu, T. C. Irving, and H. Granzier, “Titin-based modulation of calcium sensitivity of active tension in mouse skinned cardiac myocytes," Circulation Research, vol. 88, no. 10, pp. 1028-1035, 2001.

[71] N. Fukuda, Y. Wu, G. Farman, T. C. Irving, and H. Granzier, "Titin isoform variance and length dependence of activation in skinned bovine cardiac muscle," Journal of Physiology, vol. 553, no. 1, pp. 147-154, 2003.

[72] M. Krüger and W. A. Linke, "Titin-based mechanical signalling in normal and failing myocardium," Journal of Molecular and Cellular Cardiology, vol. 46, no. 4, pp. 490-498, 2009.

[73] P. F. M. van der Ven, J. W. Bartsch, M. Gautel, H. Jockusch, and D. O. Fürst, "A functional knock-out of titin results in defective myofibril assembly," Journal of Cell Science, vol. 113, no. 8, pp. 1405-1414, 2000. 
[74] H. L. Granzier, M. H. Radke, J. Peng, et al., "Truncation of titin's elastic PEVK region leads to cardiomyopathy with diastolic dysfunction," Circulation Research, vol. 105, no. 6, pp. 557-564, 2009.

[75] M. H. Radke, J. Peng, Y. Wu, et al., "Targeted deletion of titin N2B region leads to diastolic dysfunction and cardiac atrophy," Proceedings of the National Academy of Sciences of the United States of America, vol. 104, no. 9, pp. 3444-3449, 2007.

[76] N. Fukuda, Y. Wu, P. Nair, and H. L. Granzier, "Phosphorylation of titin modulates passive stiffness of cardiac muscle in a titin isoform-dependent manner," Journal of General Physiology, vol. 125, no. 3, pp. 257-271, 2005.

[77] S. Lahmers, Y. Wu, D. R. Call, S. Labeit, and H. Granzier, "Developmental control of titin isoform expression and passive stiffness in fetal and neonatal myocardium," Circulation Research, vol. 94, no. 4, pp. 505-513, 2004.

[78] Y. Wu, S. P. Bell, K. Trombitas, et al., "Changes in titin isoform expression in pacing-induced cardiac failure give rise to increased passive muscle stiffness," Circulation, vol. 106, no. 11, pp. 1384-1389, 2002.

[79] W. A. Jaber, C. Maniu, J. Krysiak, et al., "Titin isoforms, extracellular matrix, and global chamber remodeling in experimental dilated cardiomyopathy: functional implications and mechanistic insight," Circulation. Heart failure, vol. 1, no. 3, pp. 192-199, 2008.

[80] A. Borbély, I. Falcao-Pires, L. van Heerebeek, et al., "Hypophosphorylation of the Stiff N2B titin isoform raises cardiomyocyte resting tension in failing human myocardium," Circulation Research, vol. 104, no. 6, pp. 780786, 2009.

[81] I. Makarenko, C. A. Opitz, M. C. Leake, et al., "Passive stiffness changes caused by upregulation of compliant titin isoforms in human dilated cardiomyopathy hearts," Circulation Research, vol. 95, no. 7, pp. 708-716, 2004.

[82] S. F. Nagueh, G. Shah, Y. Wu, et al., "Altered titin expression, myocardial stiffness, and left ventricular function in patients with dilated cardiomyopathy," Circulation, vol. 110, no. 2, pp. 155-162, 2004.

[83] C. Neagoe, M. Kulke, F. del Monte, et al., "Titin isoform switch in ischemic human heart disease," Circulation, vol. 106, no. 11, pp. 1333-1341, 2002.

[84] Y. Wu, J. Peng, K. B. Campbell, S. Labeit, and H. Granzier, "Hypothyroidism leads to increased collagen-based stiffness and re-expression of large cardiac titin isoforms with high compliance," Journal of Molecular and Cellular Cardiology, vol. 42, no. 1, pp. 186-195, 2007.

[85] R. Yamasaki, Y. Wu, M. McNabb, M. Greaser, S. Labeit, and H. Granzier, "Protein kinase A phosphorylates titin's cardiac-specific N2B domain and reduces passive tension in rat cardiac myocytes," Circulation Research, vol. 90, no. 11, pp. 1181-1188, 2002.

[86] M. Krüger and W. A. Linke, "Protein kinase-A phosphorylates titin in human heart muscle and reduces myofibrillar passive tension," Journal of Muscle Research and Cell Motility, vol. 27, no. 5-7, pp. 435-444, 2006.

[87] M. Krüger, S. Kötter, A. Grützner, et al., "Protein kinase G modulates human myocardial passive stiffness by phosphorylation of the titin springs," Circulation Research, vol. 104, no. 1, pp. 87-94, 2009.

[88] C. Hidalgo, B. Hudson, J. Bogomolovas, et al., "PKC phosphorylation of titin's PEVK element: a novel and conserved pathway for modulating myocardial stiffness," Circulation Research, vol. 105, no. 7, pp. 631-638, 2009.
[89] B. D. Hudson, C. G. Hidalgo, M. Gotthardt, and H. L. Granzier, "Excision of titin's cardiac PEVK spring element abolishes PKC $\beta$-induced increases in myocardial stiffness," Journal of Molecular and Cellular Cardiology, vol. 48, no. 5, pp. 972-978, 2010.

[90] A. F. Leite-Moreira, P. Castro-Chaves, P. Pimentel-Nunes, et al., "Angiotensin II acutely decreases myocardial stiffness: a novel AT1, PKC and $\mathrm{Na}^{+} / \mathrm{H}^{+}$exchanger-mediated effect," British Journal of Pharmacology, vol. 147, no. 6, pp. 690-697, 2006.

[91] A. F. Leite-Moreira, C. Bras-Silva, C. A. Pedrosa, and A. A. Rocha-Sousa, "ET-1 increases distensibility of acutely loaded myocardium: a novel ETA and $\mathrm{Na}^{+} / \mathrm{H}^{+}$exchanger-mediated effect," American Journal of Physiology, vol. 284, no. 4, pp. H1332-H1339, 2003.

[92] J. S. Janicki and B. B. Matsubara, "Myocardial collagen and left ventricular diastolic dysfunction," in Left Ventricular Diastolic Dysfunction and Heart Failure, W. H. Gaash and M. M. LeWinter, Eds., pp. 3-24, Lea \& Febiger, Philadelphia, Pa, USA, 1994.

[93] G. M. Fomovsky, S. Thomopoulos, and J. W. Holmes, "Contribution of extracellular matrix to the mechanical properties of the heart," Journal of Molecular and Cellular Cardiology, vol. 48, no. 3, pp. 490-496, 2010.

[94] J. Asbun and F. J. Villarreal, "The pathogenesis of myocardial fibrosis in the setting of diabetic cardiomyopathy," Journal of the American College of Cardiology, vol. 47, no. 4, pp. 693700, 2006.

[95] M. R. Zile and D. L. Brutsaert, "New concepts in diastolic dysfunction and diastolic heart failure: part I: diagnosis, prognosis, and measurements of diastolic function," Circulation, vol. 105, no. 11, pp. 1387-1393, 2002.

[96] W. C. Little, M. R. Zile, D. W. Kitzman, W. G. Hundley, T. X. O'Brien, and R. C. Degroof, "The effect of alagebrium chloride (ALT-711), a novel glucose cross-link breaker, in the treatment of elderly patients with diastolic heart failure," Journal of Cardiac Failure, vol. 11, no. 3, pp. 191-195, 2005.

[97] Y. Sun and K. T. Weber, "Infarct scar: a dynamic tissue," Cardiovascular Research, vol. 46, no. 2, pp. 250-256, 2000.

[98] J. W. Holmes, T. K. Borg, and J. W. Covell, "Structure and mechanics of healing myocardial infarcts," Annual Review of Biomedical Engineering, vol. 7, pp. 223-253, 2005.

[99] W. J. Paulus, P. J. Vantrimpont, and A. M. Shah, "Acute effects of nitric oxide on left ventricular relaxation and diastolic distensibility in humans: assessment by bicoronary sodium nitroprusside infusion," Circulation, vol. 89, no. 5, pp. 20702078, 1994.

[100] A. M. Shah, B. D. Prendergast, R. Grocott-Mason, M. J. Lewis, and W. J. Paulus, "The influence of endotheliumderived nitric oxide on myocardial contractile function," International Journal of Cardiology, vol. 50, no. 3, pp. 225231, 1995.

[101] W. J. Paulus, "Beneficial effects of nitric oxide on cardiac diastolic function: 'the flip side of the coin,"' Heart Failure Reviews, vol. 5, no. 4, pp. 337-344, 2000.

[102] P. Castro-Chaves, R. Fontes-Carvalho, M. Pintalhao, P. Pimentel-Nunes, and A. F. Leite-Moreira, "Angiotensin IIinduced increase in myocardial distensibility and its modulation by the endocardial endothelium in the rabbit heart," Experimental Physiology, vol. 94, no. 6, pp. 665-674, 2009.

[103] C. Brás-Silva and A. F. Leite-Moreira, "Obligatory role of the endocardial endothelium in the increase of myocardial distensibility induced by endothelin-1," Experimental Biology and Medicine, vol. 231, no. 6, pp. 876-881, 2006. 
[104] C. Brás-Silva, D. Monteiro-Sousa, A. J. Duarte, et al., "Nitric oxide and prostaglandins-important players in endothelin1 induced myocardial distensibility," Physiological Research, vol. 57, no. 2, pp. 165-174, 2008.

[105] A. P. Fontes-Sousa, C. Brás-Silva, A. L. Pires, D. MonteiroSousa, and A. F. Leite-Moreira, "Urotensin II acutely increases myocardial length and distensibility: potential implications for diastolic function and ventricular remodeling," Naunyn-Schmiedeberg's Archives of Pharmacology, vol. 376, no. 1-2, pp. 107-115, 2007.

[106] A. P. Fontes-Sousa, A. L. Pires, V. F. Monteiro-Cardoso, and A. F. Leite-Moreira, "Urotensin II-induced increase in myocardial distensibility is modulated by angiotensin II and endothelin-1," Physiological Research, vol. 58, no. 5, pp. 653660, 2009.

[107] A. P. Fontes-Sousa, A. L. Pires, C. S. Carneiro, C. BrásSilva, and A. F. Leite-Moreira, "Effects of adrenomedullin on systolic and diastolic myocardial function," Peptides, vol. 30, no. 4, pp. 796-802, 2009.

[108] R. Ladeiras-Lopes, J. Ferreira-Martins, and A. F. LeiteMoreira, "Acute neurohumoral modulation of diastolic function," Peptides, vol. 30, no. 2, pp. 419-425, 2009.

[109] C. S. Apstein, "Influence of the coronary vasculature on left ventricular diastolic chamber stiffness: the erectile properties of the myocardium," in Left Ventricular Diastolic Dysfunction and Heart Failure, W. H. Gaash and M. M. LeWinter, Eds., pp. 3-24, Lea \& Febiger, Philadelphia, Pa, USA, 1994. 

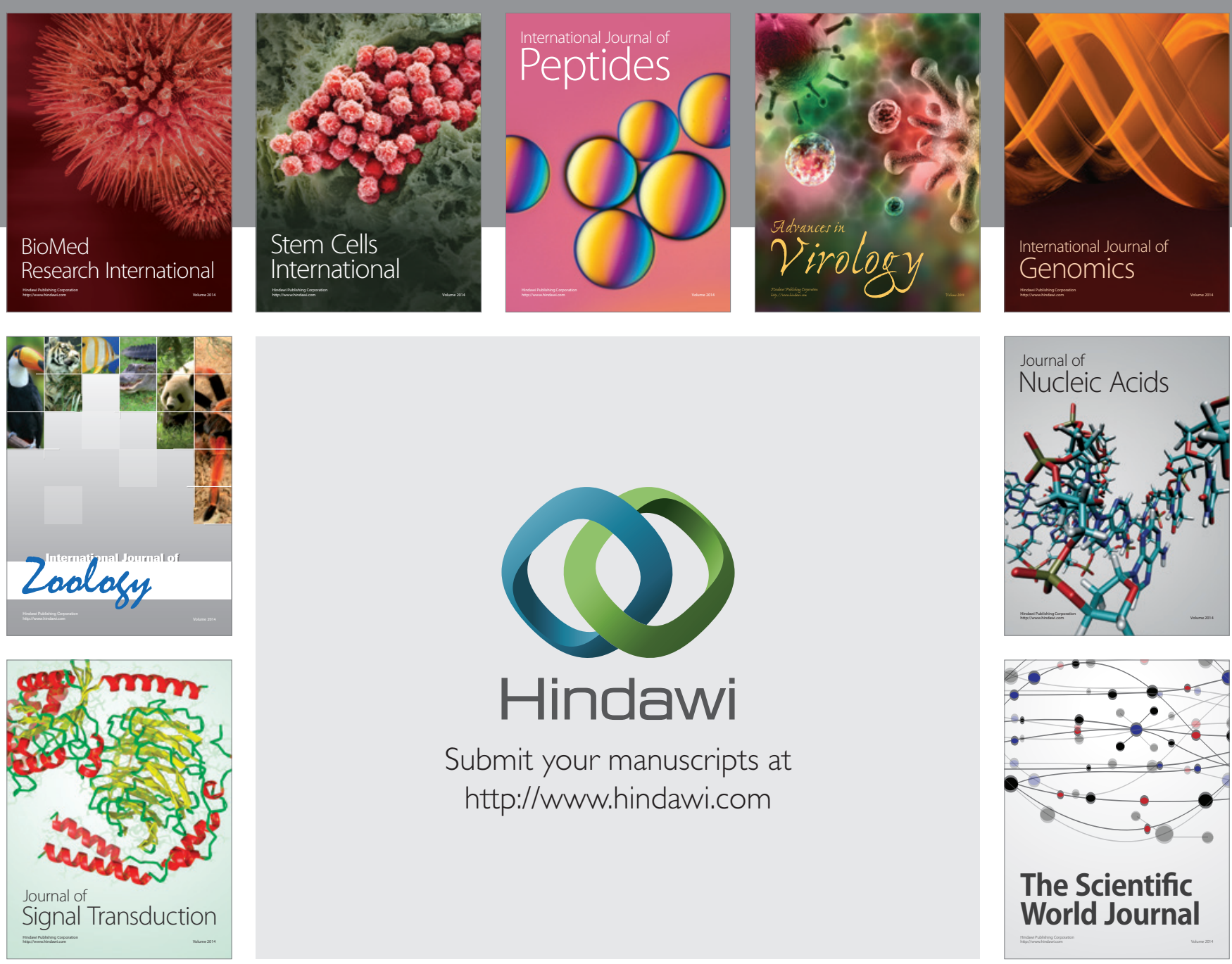

Submit your manuscripts at

http://www.hindawi.com
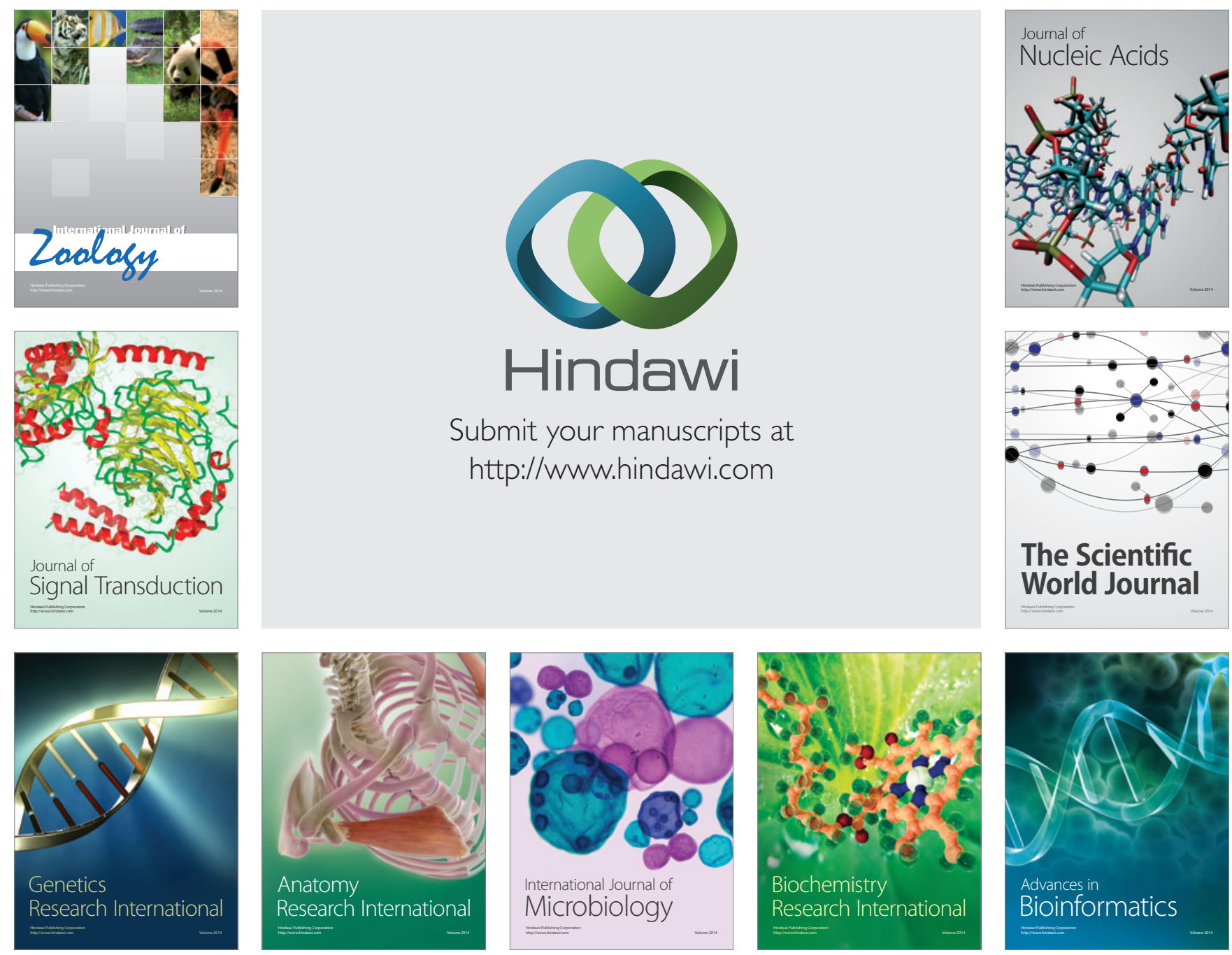

The Scientific World Journal
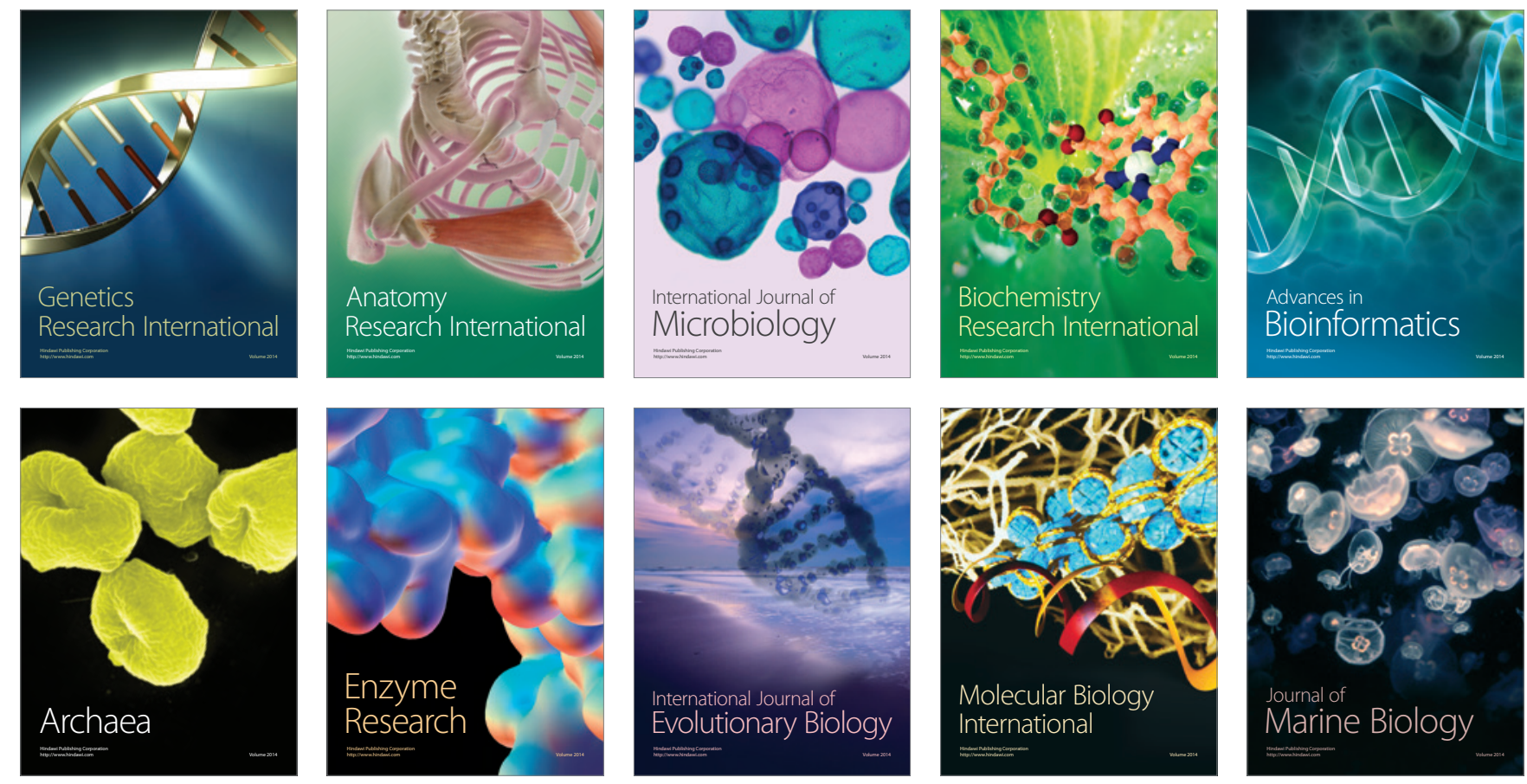